\title{
Stellar p-mode oscillations signal in Procyon A from MOST data ${ }^{\star}$
}

\author{
C. Régulo ${ }^{1,2}$ and T. Roca Cortés ${ }^{1,2}$ \\ 1 Instituto de Astrofísica de Canarias, 38205 La Laguna, Tenerife, Spain \\ 2 Dpto. de Astrofísica, Universidad de La Laguna, La Laguna, 38206 Tenerife, Spain \\ e-mail: [crr; trc]@iac.es
}

Received 28 July 2005 / Accepted 21 September 2005

\section{ABSTRACT}

Recently, Matthews et al. (2004, Nature, 430, 51) claim a null detection of p-mode oscillations in Procyon A from 32 days of nearly continuous photometric satellite-based observations (MOST). They did not even find evidence in the Fourier amplitude spectrum of Procyon of the uniform frequency spacing signature that solar-like stars acoustic spectrum must show according to asymptotic theory. However, re-analysing his data, we have found a clear evidence of signal fully compatible with what is expected from p-mode oscillations in Procyon A. Using the method developed in Régulo \& Roca Cortés (2002, A\&A, 396, 745), that allows the analysis of stellar oscillations in the acoustic power spectrum of solar-like stars when the signal is buried in noise, we have found a frequency spacing in the power spectrum of the data of $54.5 \mu \mathrm{Hz}$, that agrees well with the expectations of the theory of stellar oscillation as well as with previous estimations from ground-based observations. Moreover, our method allows the recovery of the power spectrum of the signal from the knowledge of the frequency spacing present in the data, which yields clear peaks that can be identified as acoustic p-modes. Nonetheless, as the frequency spacing coincides with the second sub-multiple of the orbital frequency of the satellite $(164.34 \mu \mathrm{Hz})$ the above findings must be taken with caution.

Key words. stars: oscillations - stars: individual: Procyon

\section{Introduction}

Procyon A, a F5 subgiant, has been considered one of the best candidates for asteroseismology due to the theoretical estimation of its p-mode amplitudes, at least $20 \mathrm{ppm}$ being possible 60 or even 70 ppm (Christensen-Dalsgaard \& Frandsen 1983; Houdek et al. 1999) which means amplitudes 5-10 times the amplitudes of the solar signals. However, ground-based observations (Brown et al. 1991; Martić et al. 1999, 2004; Eggenberger et al. 2004; Bouchy et al. 2004) reported peak amplitudes of only 2-3 times the solar ones. Even so or because of this, this star was a prime target for MOST mission (Microvariability and Oscillations of STars) a Canadian microsatellite launched on June 2003 dedicated to Asteroseismology, the detection of p-modes in Sun-like stars and detection of reflected light from exo-planets (Walker et al. 2003; Green et al. 2003).

The MOST photometry of Procyon covers 32 days with a duty cycle of $99 \%$. This impressive set of data is however contaminated by scattered Earthlight modulated by the 101.413 min orbital period of the satellite. This set of data from Procyon was firstly analysed by Matthews et al. (2004,

* Based on data from the MOST satellite, a Canadian Space Agency mission, jointly operated by Dynacon Inc., the University of Toronto Institute for Aerospace Studies and the University of British Columbia, with the assistance of the University of Vienna. hereafter M04) finding no evidence of anything other than granulation noise. Bedding et al. (2005) however, cast doubt on some of the statements of M04 paper, showing that the MOST amplitude spectrum is inconsistent with a simple noise source such as granulation. They found an excess of strong peaks in MOST amplitude spectrum that may perhaps indicate the presence of oscillations. These claims support the previous suspicion of Christensen-Dalsgaard \& Kjeldsen (2004) that MOST data are dominated by non-stellar noise.

In this work a further step is done in the analysis of MOST data that are now available to the scientific community. Using the method developed in Régulo \& Roca Cortés (2002, hereafter Paper I) it is shown that a signal fully compatible with p-mode oscillations is present in the data. From this analysis, a possible peak sequence for $l=0,1$ and 2 p-modes is obtained. In Sect. 2 an analysis of the amplitude spectrum of M04 is done followed by an analysis of their time series.

\section{Analysis of MOST photometric data}

The data used in this paper correspond to 32 days of nearly continuous photometric satellite-based observation of Procyon A. The data were obtained with MOST mission that has a telescope aperture of $15 \mathrm{~cm}$ with a broad-band optical filter (350$700 \mathrm{~nm})$. The duty cycle is of $99 \%$ with exposures of $0.9 \mathrm{~s}$ at an average rate of 6 times per minute. According to M04, 


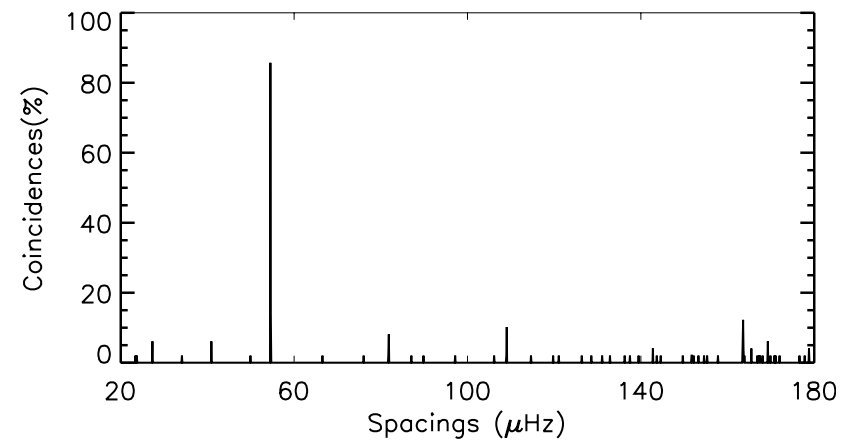

Fig. 1. Result from the search of the spacing in the power spectrum of Procyon where the frequencies of the satellite orbit and its harmonics were removed. The peak found corresponds to $54.5 \mu \mathrm{Hz}$, the expected spacing for this star.

the photometry of the instrument is somewhat contaminated by scattered Earthlight modulated by the 101.413 min orbital period of the satellite.

We have worked with the 32 days time series of the data that is shown in Fig. 2a of M04 as well as with the Fourier amplitude spectrum of Procyon shown in Fig. 3a of M04 where the frequencies of the satellite orbit and its harmonics due to modulated stray light were removed.

\subsection{Analysis of M04 amplitude spectrum}

We started with the M04 amplitude spectrum that was changed into power. This power spectrum was 8 times over sampled from the $0.36 \mu \mathrm{Hz}$ theoretical resolution of the series to $0.045 \mu \mathrm{Hz}$, having notches that correspond to the removed frequencies in a $12 \mu \mathrm{Hz}$ interval around every harmonic of the orbital period. To such power spectrum we apply the method of searching for the p-modes spectral signature developed in Paper I. What it is expected to obtain for Procyon according to the asymptotic theory for acoustic oscillations, given its known structural parameters, is a power spectrum where the p-modes acoustic frequencies are almost equally spaced at around $55 \mu \mathrm{Hz}$ in a frequency range between 0.6 and $1.4 \mathrm{mHz}$. Therefore, such frequency interval of the power spectrum is used here to search for the p-modes signature. The search is done for a range of possible frequency spacings that covers from 20 to $180 \mu \mathrm{Hz}$, yielding the results shown in Fig. 1. In the abscissa axis the spacing in $\mu \mathrm{Hz}$ is plotted and in the ordinate axis are the coincidences found in the search. Our search is repeated 50 times, changing slightly and uniformly (up to $65 \mu \mathrm{Hz})$ the range where the search is done; coincidences (in \%) means the times that the same value of the spacing is found in these 50 trials.

The peak found corresponds to $54.5 \mu \mathrm{Hz}$, in the range of spacings expected from Procyon, showing clearly the presence of signal in the data with the expected acoustic signature. This spacing is almost in the middle of previous found spacings, from the $53 \mu \mathrm{Hz}$ and $53.6 \mu \mathrm{Hz}$ found by Mosser et al. (1998) and Martić et al. (2004) respectively to the $55.5 \mu \mathrm{Hz}$ and $56 \mu \mathrm{Hz}$ found by Eggenberger et al. (2004) and Claudi et al. (2005); and it matches within the spectral resolution the $54.7 \mu \mathrm{Hz}$ obtained by Kervella et al. (2004) from their model.

As in the used power spectrum the frequencies that correspond to the satellite orbit and its harmonics have been removed, nothing appears in the search of the spacings at $v_{\text {sat }}=$ $164.3 \mu \mathrm{Hz}\left(v_{\text {sat }}^{-1}=T_{\text {orb }}=101.413 \mathrm{~min}\right)$. However, the presence of a small peak at $81.8 \mu \mathrm{Hz}$ and another at $40.9 \mu \mathrm{Hz}$ that correspond with sub-multiples of the spacing due to the orbit of the satellite is telling us that the effect of the orbit was not completely removed from the spectrum. The other three small peaks of the plot are the first sub-multiple of $54.5 \mu \mathrm{Hz}$ at $27.2 \mu \mathrm{Hz}$ and the first and second multiple at 109.0 and $163.5 \mu \mathrm{Hz}$.

Unfortunately, the spacing found for Procyon almost coincides with the second sub-multiple of the spacing due to the satellite orbit $(164.3 / 3 \approx 54.8)$. Such coincidence is especially annoying when we try to recover the original power spectrum of the data. As it was shown in Paper I, when a solar-like star has a power spectrum where the signal is buried in noise, it is possible to recover the buried signal from the knowledge of the frequency spacing $\left(v_{0}\right)$. In brief, the method works with the complex Fourier Transform of the power spectrum, by selecting only those bins spaced $v_{0}^{-1}$ and calculating the inverse Fourier Transform. The result is a recovered power spectrum of the stellar oscillations with a much better $\mathrm{S} / \mathrm{N}$ but different amplitudes or energies.

In the case of Procyon we have recovered a frequency spectrum from the one with the orbital frequency and its harmonics removed, using $54.5 \mu \mathrm{Hz}$ as the proper spacing; the frequency selected interval was from 0.6 to $1.4 \mathrm{mHz}$. A clear set of peaks appear in the spectrum, see Fig. 2. They show a distribution that could be interpreted as what it is expected for the $l=0,1$ and $2 \mathrm{p}$-modes, according with the asymptotic theory. However some other peaks appear that we could not match with any expected p-mode series, based on the previous identification. For clarity, a piece of this recovered spectrum, from 0.925 to $1.1035 \mathrm{mHz}$, is shown in Fig. 3, where the found peaks have been marked as $l=A, B, C, D, E$. This is the pattern that is found repeated all along the recovered spectrum. A possible identification of these peaks could be $A=l=1, C=l=2$, $D=l=0$ although this is not the only one. According to the frequency identifications of Martić et al. (2004) and Eggenberger et al. (2004), $l=A$ peaks could correspond to $l=1 \mathrm{p}$-modes and $l=D$ to $l=2$. Moreover, some frequencies of the sets of peaks we have matched as $l=B$ are close to some of the frequencies that Kervella et al. (2004) obtain from their model for the $l=2 \mathrm{p}$-modes and some $l=C$ are near to the $l=0$ p-modes calculated from the model. Even more puzzling, the peaks $l=B$ have a frequency separation of $l=A$ and $l=D$ of $\pm 11.7 \mu \mathrm{Hz}$, a frequency that could correspond to a $24 \mathrm{~h}(11.6 \mu \mathrm{Hz})$ modulation of the signal, being the $l=E$ peaks at the same distance, $11.7 \mu \mathrm{Hz}$, from the $l=D$ peaks. Although the satellite signal has not a $24 \mathrm{~h}$ window function, maybe daily changes could exist in the signal due to scattered Earthlight.

It is important to remark that, if the recovery procedure of the frequency spectrum is done with $54.8 \mu \mathrm{Hz}\left(v_{\mathrm{sat}} / 3\right)$, the recovered spectrum does not show clear peaks above the noise. Moreover, although we could not establish any relation 


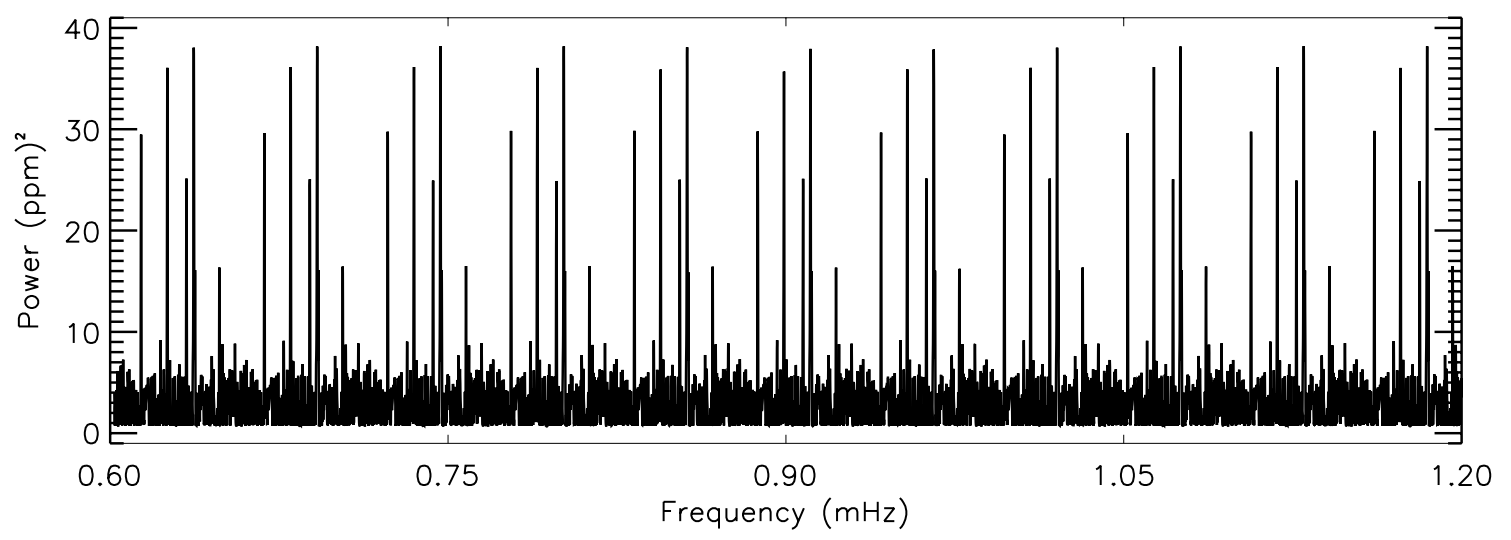

Fig. 2. Recovered power spectrum of Procyon using the method developed in Régulo \& Roca Cortés (2002).

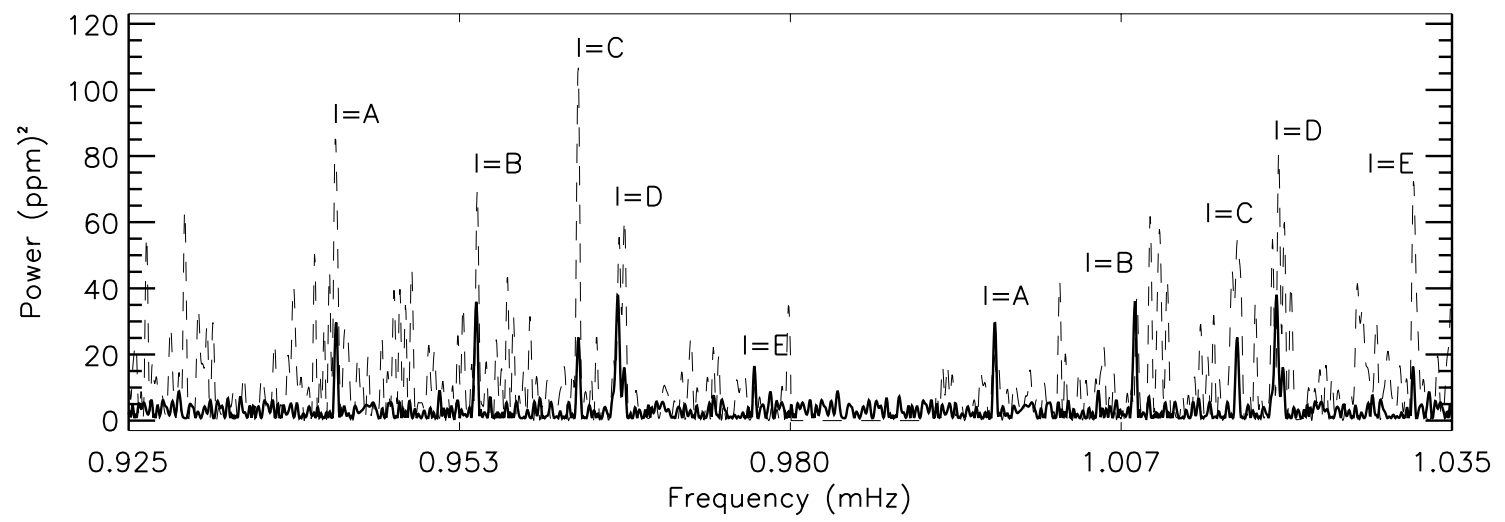

Fig. 3. A piece of the recovered power spectrum of Procyon shown in Fig. 2. The recovered power spectrum (thick line) is plotted over the original one (dashed line) for comparison.

between any recovered peak and the orbital period or its harmonics and even using the power spectrum where this orbital period and its harmonics have been removed, it is not possible to reject the possibility that some not well removed part of such instrumentally driven period could introduce spurious signal in the recovered spectrum.

\subsection{Analysis of M04 time series}

To see how things appear when the orbital effect is not removed, the time series of data has been also analysed. The first step was to remove the slow trend that the data show, subtracting a second order polynomial from the time series. The second step was to obtain the power spectrum. This was done using an iterative sine wave fitting procedure instead of a Fast Fourier Transform since the time series is not equally spaced in time. The method consists of fitting a simple sine wave function $f(t)=\alpha \sin (2 \pi v t)+\beta \cos (2 \pi v t)$ at any given $v$ to the data. From $\alpha$ and $\beta$, the amplitude and phase of the fitted function is obtained. The scanned frequency range covers from 0.2 to $1.8 \mathrm{mHz}$ in steps of $0.1 \mu \mathrm{Hz}$. In this power spectrum that now contains the spurious effect due to the diffuse light combined with the orbital period, the same search for the spacing used with the previous power spectrum was done and the result is shown in Fig. 4. The highest peak (39\%) corresponds now to the frequency spacing of the harmonics of the satellite orbit $(164.3 \mu \mathrm{Hz})$ and the second one at $54.8 \mu \mathrm{Hz}(35 \%)$ is the

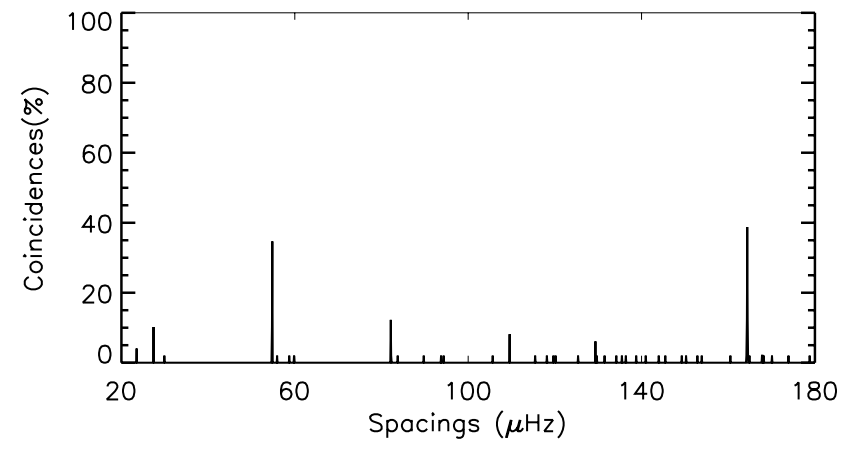

Fig. 4. Result from the search of the spacing in the power spectrum of Procyon where the frequencies of the satellite orbit and its harmonics have not been removed. The two highest peaks from left to right correspond, in $\mu \mathrm{Hz}$, to: 54.8 (the spacing of the star) and 164.3 (the spacing due to the satellite orbit).

spacing of the star. Within the frequency resolution of the spectrum $(0.36 \mu \mathrm{Hz})$ is the same bin than the obtained before $(54.5 \mu \mathrm{Hz})$; probably the small shift is due to the fact that now the spacing of the star is mixed with the sub-multiple, $v_{\text {sat }} / 3$, of the orbital frequency of the satellite. The method of searching for the spacing also finds the multiples and submultiples of any frequency spacing present; in fact the small peaks that appears in the plot (Fig. 4) are the multiple and submultiple of $54.8 \mu \mathrm{Hz}$ at $109.6 \mu \mathrm{Hz}$ and at $27.4 \mu \mathrm{Hz}$ as well as the sub-multiple of $v_{\mathrm{sat}}=164.3 \mu \mathrm{Hz}$ at $82.1 \mu \mathrm{Hz}$. The level of 
coincidences in this second search is smaller than in the previous one (Fig. 1), probably because now two frequency spacings are present in the power spectrum, one being very close (at the same bin) than the sub-multiple $v_{\text {sat }} / 3$ of the other.

Another way of cleaning the photometric set of data of the influence of the satellite's orbit on the instrument was tried. Using a phase analysis, modulo $T_{\text {orb }}=101.41 \mathrm{~min}$, the influence on the instrumental noise was searched for. A clear periodicity of $25 \mathrm{ppm}$ and $11 \mathrm{ppm}$ amplitude at $T_{\text {orb }}$ and $T_{\text {orb }} / 2$ is found to be present in the set of data as well as a peak $120 \mathrm{ppm}$ tall and 2 min wide. The rest of harmonics of $T_{\text {orb }}$ are less than 6 ppm amplitude. Therefore, we have "cleaned" the data by subtracting such average behaviour of the instrumental noise, representing $\sim 0.01 \%$ of the $\sigma$ of the data, and repeating the whole procedure. Obviously, the power spectrum at $v_{\text {sat }}$ and $2 v_{\text {sat }}$ has now diminished but their effect at other frequencies is almost negligible. The results yielded by the search change very little from what has been found in this subsection.

\section{Discussion and conclusions}

The 32 days of nearly continuous photometric observations of Procyon obtained with MOST satellite have been analysed, using the method developed in Paper I, to search for stellar acoustic oscillations in the power spectrum of solar-like stars.

This set of data was previously analysed by the MOST scientific group (M04) finding no evidence of acoustic signal but only granulation noise. Moreover, in a recent letter Bedding et al. (2005)'s analysis of the same data yield the conclusion that an acoustic excess of power exists but buried in the granulation noise. In this paper, we have found clear evidence of the signature of p-modes with a frequency spacing of $v_{0}=54.5 \mu \mathrm{Hz}$.

Furthermore, a power spectrum of the signal is recovered, where a clear set of peaks appears being possible to identify some of them as acoustic p-modes. Unfortunately, we can not exclude the possibility of having spurious signal in the recovered spectrum due to the nasty coincidence between the sub-multiple $v_{\text {sat }} / 3$ of an instrumental signal generated by the orbital period of the satellite and the Procyon's frequency spacing of its acoustic oscillations proposed here.

Given the interest of having a good photometric observation of Procyon A to compare with spectroscopic observations, it would be very interesting to have another run with MOST when the satellite looses some hight and the orbit period diminishes by some $5 \%$.

Acknowledgements. This work has been partially funded under grants AYA 2001-1571 and ESP 2004-03855-C03-03 of the Spanish national research plan. We thank the referee for useful comments which improved the paper.

\section{References}

Bedding, T. R., Kjeldsen, H., Bouchy, F., et al. 2005, A\&A, 432, L43 Bouchy, F., Maeder, A., Mayor, M., et al. 2004, Nature, 432, 7015

Brown, T. M., Gilliland, R. L., Noyes, R. W., \& Ramsey, L. W. 1991, ApJ, 368, 599

Chistensen-Dalsgaard, J., \& Frandsen, S. 1983, Sol. Phys., 82, 469

Chistensen-Dalsgaard, J., \& Kjeldsen, H. 2004, Nature, 430, 29

Claudi, R. U., Bonanno, A., Leccia, S., et al. 2005, A\&A, 429, L17

Eggenberger, P., Carrier, F., Bouchy, F., \& Blecha, A. 2004, A\&A, 422, 247

Green, D., Matthews, J. M., Seager, S., \& Kuschinig, R. 2003, ApJ, 597,590

Houdek, G., Balmforth, N. J., Chistensen-Dalsgaard, J., \& Gough, D. O. 1999, A\&A, 351, 582

Kervella, P., Thévenin, F., Morel, P., et al. 2004, A\&A, 413, 251

Martić, M., Schmitt, J., Lebrun, J.-C., et al. 1999, A\&A, 351, 993

Martić, M., Lebrun, J.-C., Appourchaux, T., \& Korzennik, S. G. 2004, A\&A, 418, 295

Matthews, J. M., Kusching, R., Guenther, D. B., et al. 2004, Nature, 430, 51

Mosser, B., Maillard, J. P., Mékarnia, D., \& Gay, J. 1998, A\&A, 340, 457

Régulo, C., \& Roca Cortés, T. 2002, A\&A, 396, 745

Walker, G., Matthews, J., Kuschnig, R., et al. 2003, PASP, 115, 1023 